\title{
The study of a CVT belt using the composite material of carbon fiber-reinforced thermoplastic resin
}

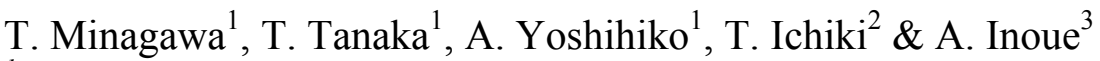 \\ ${ }^{I}$ Graduate School of Doshisha University, Japan \\ ${ }^{2}$ Bando Chemical Industries, LTD, Japan \\ ${ }^{3}$ Toyo Machinery and Metal Co Ltd, Japan
}

\begin{abstract}
The purpose of this study is to develop automotive frictional parts, especially continuously variable transmission (CVT) blocks by using a composite material made of thermoplastics resin and fiber. CVT blocks are used at about $100^{\circ} \mathrm{C}$ under high pressure and friction condition. So, we have used nylon 9T, PA9T. PA9T has high a melting point that is higher than maximum temperature of the state of slip. To apply composite materials to CVT blocks, the material properties such as high strength, modulus of elasticity and fatigue strength are important. Therefore, a purpose of this study is improvements of mechanical properties by adding fillers to PA9T with various compounding and molding condition. PA9T and CF were mixed by using twin screw extruder. After compounding, dumbbell specimens were made by using injection molding machine. Bending, fatigue and abrasion tests were conducted. Moreover, we evaluated the transmission capacity of CVT belt made of PA9T composites and conducted slip test. According to results of tests, PA9T with CF38.8 vol\% achieved desired values of CVT. The CVT belt has $40 \mathrm{Nm}$ of torque under $2.0 \mathrm{kN}$ of axle load.

Keywords: CVT, PA9T, transmission capacity evaluating test.
\end{abstract}

\section{Introduction}

In recent years, global warming, the deplete of fossil fuel, and the problem of waste are being closed up. Therefore, manufacturing with a consideration of an environment is important. Thermoplastics resin becomes a liquid by heating above its melting temperature and a solid when it is cooled. Accordingly, it is 
easy to cast and recycle thermoplastics resin. In addition, applying the plastics resin instead of metal leads to an improvement of the fuel efficiency of a car by reducing the weight of it. Therefore, in this study, we aim at developing automotive frictional parts using carbon fiber-reinforced thermoplastic resin, especially CVT (continuously Variable Transmission) blocks by using a composite material made of thermoplastics resin and fiber. CVT blocks are rubbed at about $100^{\circ} \mathrm{C}$ under high pressure condition. So, we have used nylon 9T, PA9T as base material of thermoplastics resin [1]. PA9T has high a melting point $\left(304^{\circ} \mathrm{C}\right)$ that is higher than maximum temperature of the state of slip and high strength in plastics. To apply composite materials to CVT blocks, the states of being high strength, modulus of elasticity and fatigue strength are important. Therefore, a purpose of this study is improvements of strength, modulus of elasticity and fatigue strength by adding fillers to PA9T with various compounding and molding condition. PA9T and CF were mixed by using twin screw extruder. After compounding, dumbbell specimens were made by using injection molding machine. Bending and fatigue tests were conducted. We also conducted abrasion test because CVT blocks are rubbed under high pressure. Moreover, we evaluated a transmission capacity evaluating [2-5] and slip test of belt in order to confirm whether or not mechanical properties of PA9T composites satisfy desired values CVT blocks.

\section{Experiments}

\subsection{Specimen}

In this study, N1000A (Normal grade) and N1001A (Antifriction grade) of PA9T (Kuraray-made, Genestar ${ }^{\mathrm{TM}}$ ) were used. Carbon fiber, CF (Mitsubishi Rayon-made, TR06NE) and Boron nitride, BN (Denki Kagaku Kogyo-made, Powder GP) was used as the filler. Table 1 shows filler contents of pellets. PA9T and CF were mixed by using twin screw extruder (coperion-made, ZSL18). After compounding, dumbbell specimens were made by using injection molding machine (Toyo Machinery and Metal-made, Plaster ET-40V). Each pellets were dried in an oven under $80^{\circ} \mathrm{C}$ during $24 \mathrm{~h}$ before mixing and molding. Table 2 shows molding condition.

Table 1: Fabrication condition of pellets.

\begin{tabular}{|c|c|c|c|c|c|}
\hline Matrix & $\mathrm{CF}(\mathrm{vol} \%)$ & $\begin{array}{c}\mathrm{BN} \\
(\mathrm{vol} \%)\end{array}$ & Matrix & $\mathrm{CF}(\mathrm{vol} \%)$ & $\begin{array}{c}\mathrm{BN} \\
(\mathrm{vol} \%)\end{array}$ \\
\hline N1000A & 0 & 0 & N1001A & 0 & 0 \\
\hline & 13.7 & 0 & & 13.7 & 0 \\
\hline & 29.7 & 0 & & 29.7 & 0 \\
\hline & 38.8 & 0 & & 38.8 & 0 \\
\hline & 48.7 & 0 & & 48.7 & 0 \\
\hline & 41.2 & 7.0 & & - & - \\
\hline
\end{tabular}


Table 2: Mixing and injection condition.

\begin{tabular}{ccc}
\hline Machine & Setting conditions & \\
\hline twin screw extruder & Screw rotation speed $[\mathrm{rpm}]$ & 150 \\
& Temperature $\left[{ }^{\circ} \mathrm{C}\right]$ & 330 \\
injection molding machine & Screw rotation speed $[\mathrm{rpm}]$ & 150 \\
& Injection pressure $[\mathrm{MPa}]$ & 150 \\
& Back pressure $[\mathrm{MPa}]$ & 3.0 \\
& Holding pressure $[\mathrm{MPa}]$ & 50 \\
Cylinder temperature $\left[{ }^{\circ} \mathrm{C}\right]$ & 330 \\
Injection speed $[\mathrm{mm} / \mathrm{s}]$ & 50 \\
& Holding time $[\mathrm{s}]$ & 10 \\
Cooling time $[\mathrm{s}]$ & 15 \\
& Mold temperature $\left[{ }^{\circ} \mathrm{C}\right]$ & 120 \\
\hline
\end{tabular}

\subsection{Evaluation of mechanical properties}

Bending and fatigue test were conducted to examine whether PA9T has sufficient strength of CVT belt or not. Fiber length and dispersion are measured to investigate effects of molding process on them.

Dumbbell specimen was used for the measurement of fiber length. It was cut $4 \mathrm{~mm}$ from the center, the resin was evaporated by heating, residual fibers were dispersed in the water and fiber length was measured by optical microscope. 1000 fibers were measured in each condition.

Center of dumbbell specimen was cut and the cross-section was polished and observed by optical microscope in measurement of dispersion. After fibers and resin were color-coded in the photograph, it was decided into $n \times n$ bars. Each lattices of area rates were measured and standard deviations were calculated. Moreover, fractal value that the inclination of double-logarithmic graph of standard deviations of area rates with $n$ times -1 was used as index of dispersion. Fractal value is from 0 to 1 and Close to 1 means good dispersion.

Three-point bending tests were conducted under the testing rate $2.0 \mathrm{~mm} / \mathrm{s}$ and span length of $64 \mathrm{~mm}$. And the ambient temperature is $120^{\circ} \mathrm{C}$ by using a high temperature oven. Desired values are bending strength $200 \mathrm{MPa}$ and modulus $20 \mathrm{GPa}$.

Fatigue tests were conducted at room temperature. Frequency of the test was controlled as $5.0 \mathrm{~Hz}$. Desired values are fatigue strength $60 \mathrm{MPa}$ at $10^{7}$ times.

Ring-on-disc abrasion tests were conducted under axial loading of $1.2 \mathrm{kN}$ and sliding speed $50 \mathrm{rpm}$. Counter materials were $\mathrm{S} 45 \mathrm{C}$ and $\mathrm{Ra}=0.2[\mu \mathrm{m}]$. Fig. 1 and 2 shows the shapes of a specimen and a counter material. 


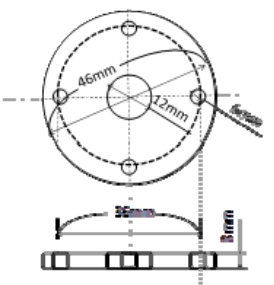

Figure 1: Specimen of abrasion test. Figure 2: Counter material of abrasion test.

\subsection{Assessments of abilities of CVT belt}

The transmission capacity evaluating and slip tests were conducted to evaluate PA9T belt. Fig. 3 shows schematic of testing machine. Each testing methodology is shown below.

Evaluation of the transmission capacity was conducted to measure the allowable transmitting torque. The test was conducted under tension between pulleys of 980,1176,1372, 1568, 1764 and $1960 \mathrm{~N}$ and rotating speed of $5500 \mathrm{rpm}$. The allowable transmitting torque and the temperature of belt were measured by using torquemeter and thermoviewer as driving side torque was increase gradually until slip was occured. The allowable transmitting torque is a driving side torque at slip rate of $1.4 \%$. The desired value of transmission capacity is $40 \mathrm{Nm}$. And a running-in were conducted a hour under tension between pulleys $0 \mathrm{~N}$ and rotating speed 5,500 rpm before test to accustom contact area to wear.

The slip test was conducted to evaluate that whether CVT belt can make a comeback to normal condition after slipping of belt. The test was conducted under tension between pulleys of 980,1176, 1372, 1568, 1764 and $1960 \mathrm{~N}$, rotating speed 5,500 rpm, driving side torque $40 \mathrm{Nm}$, and whether CVT belt can make a comeback to normal condition after slipping were checked. The tension between pulleys was increased from $980 \mathrm{~N}$ until the melting of CVT belt was confirmed.

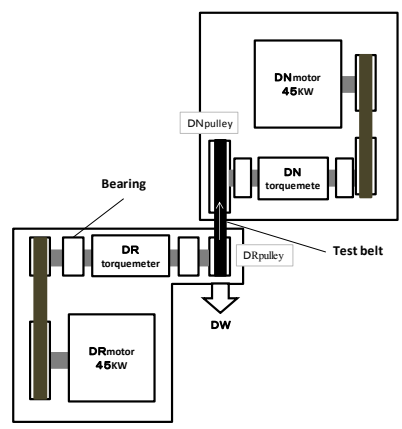

Figure 3: Testing machine of evaluation of CVT belt. 


\section{Results and discussion}

\subsection{Measurement of fiber length and dispersibility}

The fiber length and dispersibility of pellets and specimen were measured. The initial fiber length of $\mathrm{CF}$ is $6 \mathrm{~mm}$. Table 3 shows results of fiber length and dispersibility. According to Table 3, fiber length of N1000ACF48.7 vol\% was decreased by $85 \%$ after compounding of twin-screw extruder. And by molding using standard or $\mathrm{V}$ and $\mathrm{D}$ screw, fiber length of specimen was decrease by $41 \%$ and $30 \%$ compared to pellets, respectively. V and D screw is the screw that can reduce the fiber break during plasticization in injection process. It included dulmadge zone to improve fiber dispersion. Therefore, the fiber length was shorten by high shear at the Dulmadge zone after melting. In terms of dispersibility, the $\mathrm{V}$ and $\mathrm{D}$ screw was better than standard screw because high shear stress was taken.

Table 3: Results of fiber length and dispersibility.

\begin{tabular}{cc|ccc}
\hline Materials & CF content[vol\%] & Fractal Value & $\begin{array}{c}\text { Fiber length of } \\
\text { Pellets[mm] }\end{array}$ & $\begin{array}{c}\text { Fiber length of } \\
\text { specimen[mm] }\end{array}$ \\
\hline \multirow{3}{*}{ N1000A } & 13.7 & 0.73 & 0.94 & 0.71 \\
& 29.7 & 0.81 & 0.90 & 0.68 \\
VandDN1000A & 38.8 & 0.84 & 0.88 & 0.62 \\
& 48.7 & 0.83 & 0.83 & 0.61 \\
N1001A & 38.8 & 0.90 & 0.99 & 0.58 \\
\hline & 13.7 & 0.71 & 0.92 & 0.70 \\
& 29.7 & 0.79 & 0.89 & 0.67 \\
& 38.8 & 0.83 & 0.88 & 0.63 \\
\end{tabular}

\subsection{Bending test}

Fig. 4 shows results of bending test in at $120^{\circ} \mathrm{C}$. According to Fig. 4 , bending strength and modulus increased as $\mathrm{CF}$ contents increase. Bending strength and modulus were less about $20 \%$ than desired value that is bending strength of $200 \mathrm{MPa}$ and modulus of $20 \mathrm{GPa}$ in the case of CF38.8 vol\%. Bending strength and modulus were better about $2 \%$ and $20 \%$ than desired value in case of CF48.7 vol\% respectively. Bending strength and modulus were not improved by using $\mathrm{V}$ and $\mathrm{D}$ screw. And bending strength and modulus of N1000ACF38.8 vol\% increased by $33 \%$ and $21 \%$ and were better $7 \%$ and $2 \%$ than desired value by annealing respectively. Annealing treatment is $2 \mathrm{~h}$ under $150^{\circ} \mathrm{C}$. For the specimen without annealing, the mold temperature is controlled at $120^{\circ} \mathrm{C}$. The crystallization temperature of this material is $130^{\circ} \mathrm{C}$. Therefore, annealing at $150^{\circ} \mathrm{C}$ increase crystallization of the specimen. 
182 High Performance and Optimum Design of Structures and Materials

Table 4: Results of bending strength and elastic modulus.

\begin{tabular}{cc|cc}
\hline Materials & CF content[vol\%] & Bending strength[MPa] & Bending modulus[GPa] \\
\hline & 0 & 26.2 & 1.3 \\
N1000A & 13.7 & 51.1 & 3.8 \\
& 29.7 & 139.3 & 11.5 \\
& 38.8 & 162.1 & 16.9 \\
VandDN1000A & 48.7 & 204.0 & 24.0 \\
AnealingN1000A & 38.8 & 160.0 & 17.0 \\
& 38.8 & 215.7 & 20.4 \\
\hline \multirow{2}{*}{ N1001A } & 0 & 30.2 & 1.3 \\
& 13.7 & 45.2 & 2.9 \\
& 29.7 & 125.6 & 10.6 \\
& 38.8 & 162.1 & 23.8 \\
\hline
\end{tabular}

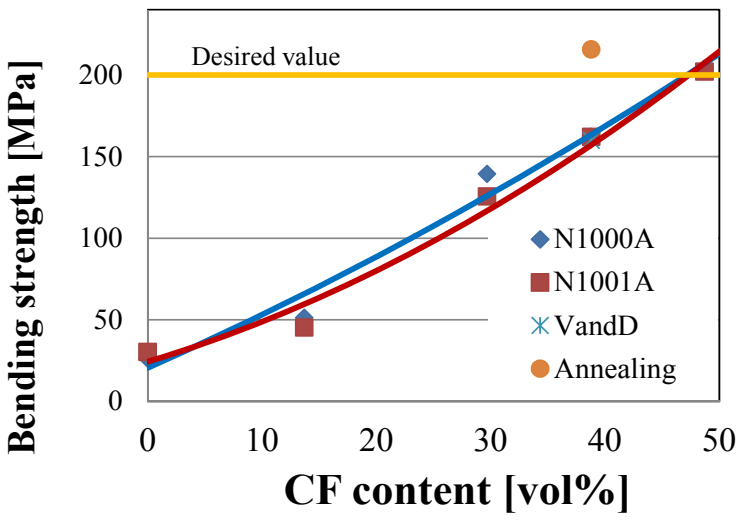

(a)

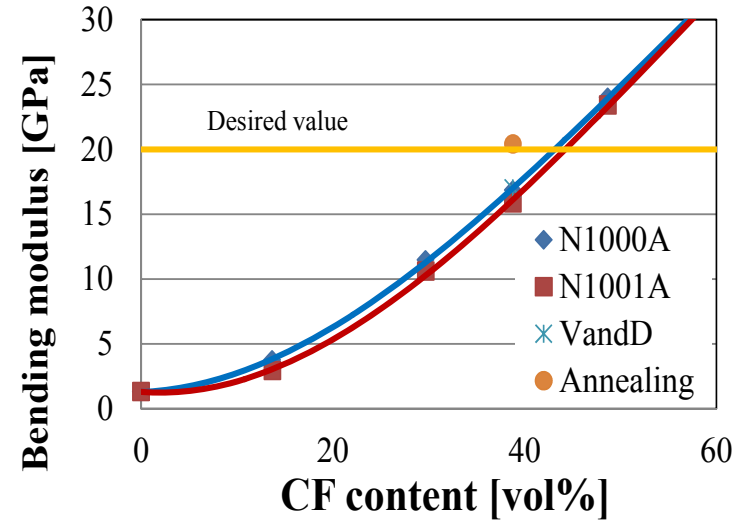

(b)

Figure 4: Results of bending strength and elastic modulus: (a) Bending strength with CF content, (b) bending modulus with CF content. 


\subsection{Fatigue test}

Fatigue tests of N1000ACF38.8 vol\% and 48.7 vol\% were conducted. Fig. 5 shows results of fatigue tests. According to Fig. 5, N1000ACF38.8 vol\% and AnnealingN1000ACF38.8 vol\% of fatigue strength exceeded desired value. However N1000ACF 48.7 vol\% of fatigue strength decreased. The reason for this is the stress concentration by shaped precision and increase fiber.

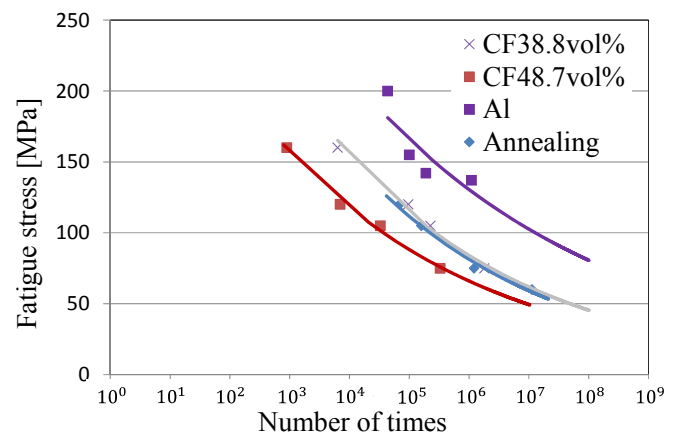

Figure 5: Results of Fatigue test.

\subsection{Friction abrasion test}

The friction abrasion test of N1000ACF38.8 vol\% and N1001ACF38.8 vol\% were conducted. Fig. 6 shows results of friction abrasion test. According to Fig. 6 , abrasion wear of N1000A (Normal glade) was less than N1001A (Antifriction grade). Wear properties of N1000A is investigated. And because abrasion wear larger than desired value, BN was mixed to modify abrasion wear of N1000ACF38.8 vol\%. Abrasion wear attained desired value by adding BN7 vol\%. And BN decrease not only abrasion wear but also surface temperature by improving wear properties and thermal conductivity [6-10].

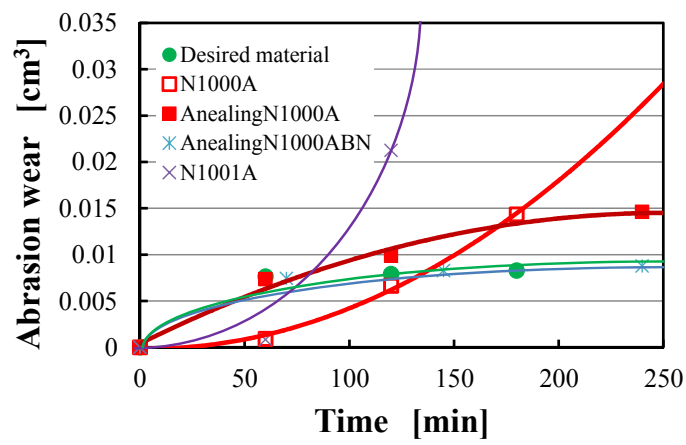

Figure 6: Results of friction abrasion test with CF38.8 vol\%. 


\section{Evaluation of CVT belt}

In Section 3, the desired values were attained in bending and fatigue tests. So, CVT belt was molded actually and the properties were evaluated. The molding of CVT belt is that the gum was hold between blocks that were used 200 pieces after injection molding of CVT blocks. Fig. 7 shows a shape of CVT block. The used materials were N1000ACF38.8 vol\%(N1000ACF) and N1000ACF41.2 vol\% BN7 vol\%(N1000ACFBN). Results and discussion of each test are shown below.

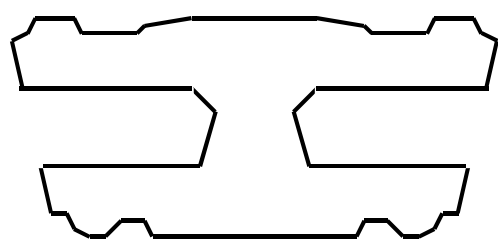

Figure 7: Shape of CVT block.

\subsection{Transmission capability test}

Fig. 8 shows results of transmission capability test. Fig. 8 is graph of slip rate with driving side torque, slip rate increases linearly at the start and non-linearly at the certain driving side torque. An allowable torque is a maximum torque in a linear range. N1000ACF and N1000ACFBN attained transmission torque of $40 \mathrm{Nm}$ under tension between pulleys of $1960 \mathrm{~N}$. The transmission torque of N1000ACFBN was slightly better than N1000ACF. Because CVT belt transfers power by frictional resistance at contact surface, transmission capability becomes high when friction coefficient becomes high. However friction coefficient of N1000ACFBN was less than N1000ACF. For searching this cause, the contact surfaces were observed. the worn marks were observed merely at the contact surfaces of upper beam of both materials. For searching this cause, an angle of $\mathrm{V}$ of CVT belt was measured. Angles of V of pulley and CVT block are $26^{\circ}$. Table 5 shows the angle, thickness and thermal conductivity of blocks. According to Table 5, the V angle of N1000ACFBN was similar to $26^{\circ}$ compared to N1000ACF. Therefore the contact area got larger and communicative competence got higher. The reason of this is fill of upper beam. According to Table 5, thickness of the upper beam of N1000ACFBN is larger than N1000ACF. A counterforce that accrues by holding gum between upper and under beam pushes up upper beam. As a thickness of upper beam becomes large, a hardness becomes large and upper beam can prevent bending. Therefore $\mathrm{V}$ angle can retain near $26^{\circ}$. So the transmission torque of N1000ACFBN is better than N1000ACF.

Then, temperatures of belts are discussed. Fig. 9 shows temperatures of CVT belts on transmission capability test. According to Fig. 9, temperature of belt was about $49^{\circ} \mathrm{C}$ at the driving side torque $40 \mathrm{Nm}$. The melting of contact areas were not observed after tests. 
Table 5: Angle, thickness and thermal conductivity of blocks.

\begin{tabular}{c|cc}
\hline & N1000ACF & N1000ACFBN \\
\hline Angle of $\mathrm{V}\left[^{\circ}\right]$ & 25.2 & 25.8 \\
Thickness of uneder beam $[\mathrm{mm}]$ & 2.45 & 2.50 \\
Thickness of upper beam $[\mathrm{mm}]$ & 2.96 & 3.13 \\
Thermal conductivity $[\mathrm{W} / \mathrm{mK}]$ & 0.893 & 1.384 \\
\hline
\end{tabular}

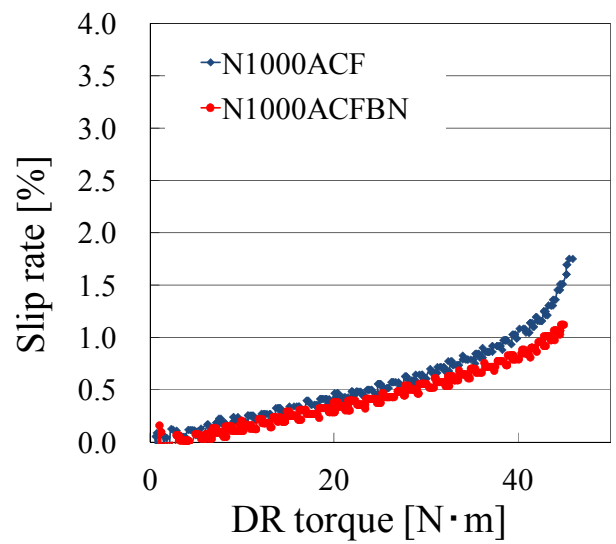

Figure 8: Transmission capability under tension between pulleys of 1960N.

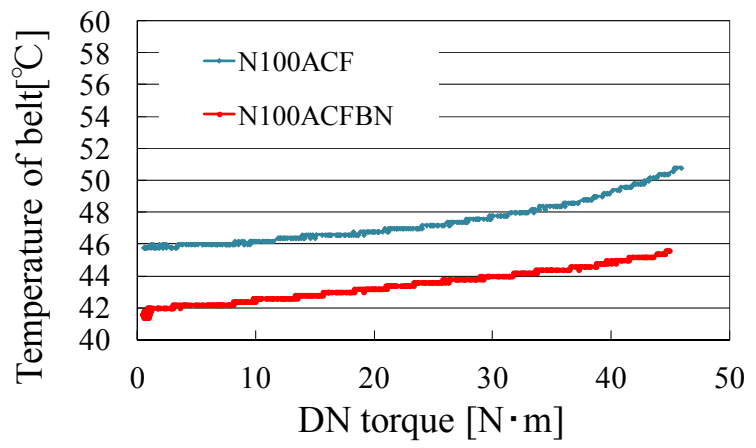

Figure 9: Temperature of CVT blocks on transmission capability test under tension between pulleys of $1960 \mathrm{~N}$.

\subsection{Slip test}

Fig. 10 and 11 show results of slip tests. Point 1 is contact area of blocks at the exit driving side pulley and Point 2 is contact area of blocks at the portal driving side pulley. According to Fig. 10, both materials couldn't return to normal 
condition after slipping. Both materials couldn't clear the slip test. In the case of $\mathrm{N} 1000 \mathrm{ACF}$, resin melted in first test. In the case of composite with BN, it passed two times of slip test, and then melted after three times. And a slip could be prevented to mix BN. The reason of this is that $\mathrm{V}$ angles become nearly $26^{\circ}$, contact between pulley and belt becomes better as mentioned at 4.1. According to Fig. 11(b), a slope of slip rate became smaller as number of test became more. This cause is that $\mathrm{V}$ angle become nearly $26^{\circ}$ because of wear of resin. Then the cause of melting is high temperature because of generation of wear heat. According to Fig. 11, maximum temperature decrease by $34 \%$ by mixing BN. This cause is improvement of not only friction coefficient but also thermal conductivity. According to Table 5, thermal conductivity increases by $55 \%$ by mixing BN7 vol\%. The temperature of contact area decreases because the heat moves from contact area to center of CVT blocks due to improvement of thermal conductivity. Moreover because other surfaces are larger than contact are, heat transfer to the air became lager by moving the heat to the whole of block and the total temperature of CVT block decrease. Therefore the slip property was improved by adding BN.

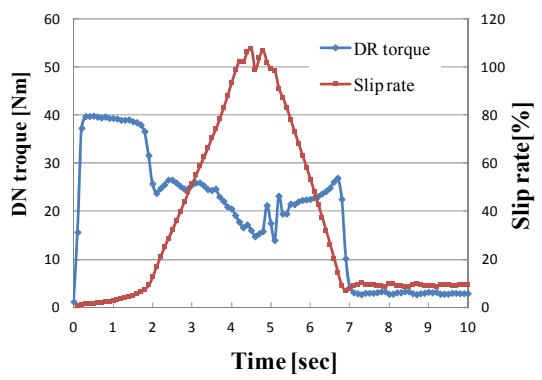

(a) $\mathrm{N} 1000 \mathrm{ACF}$

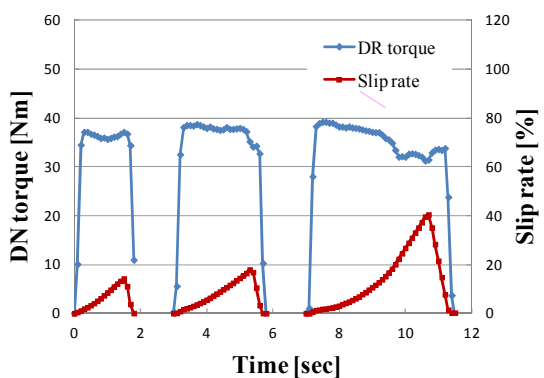

(b) N1000ACFBN

Figure 10: Results of slip tests.

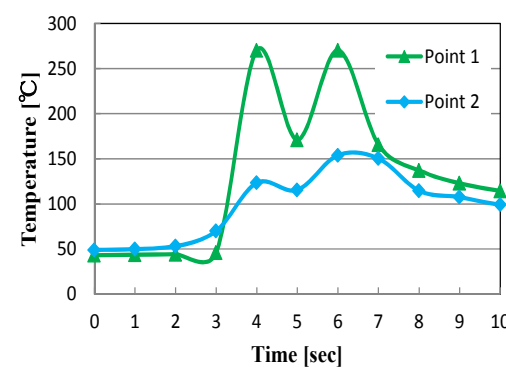

(a) N1000ACF

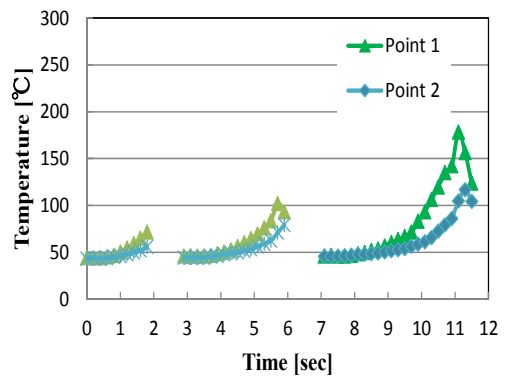

(b) N1000ACFBN

Figure 11: Temperature of CVT blocks on slip test. 


\section{Conclusion}

There were no differences of mechanical properties between N1000ACF38.8 vol\% and N1001ACF38.8 vol\%. PA9TCF38.8 vol\% attained desired values of mechanical properties of CVT block. Because the frictional property of N1000A with CF38.8 vol\% was better than N1001A with CF38.8 vol\%, N1000A use to CVT blocks. N1000ACF38.8 vol\% and N1000ACF41.2 vol\% BN7 vol\% attained transmission torque $40 \mathrm{Nm}$ under tension between pulleys of $1960 \mathrm{~N}$. And the melting on the slip came to hardly occur by adding BN7 vol\% because the surface temperature was relieved by improving abrasion resistance and thermal conductivity.

\section{Acknowledgements}

The authors thank to Mr. Katsuhiko Hata and Katuyosi Fujiwara of Bando Chemical Industries, Ltd. for their advice and Mr. Keiichiro Matsuo and Ms. Yukiko Shirai of Bando Chemical Industries, Ltd. for their help in this work. And this work was financially supported by the Program for the Strategic Research Foundation at Private Universities, 2013-2017, MEXT of Japan and Adaptable and Seamless Technology Transfer Program through Target-driven R\&D, Japan Science and Technology Agency.

\section{References}

[1] Kuraray Genestar ${ }^{\mathrm{TM}}$, http://www.genestar.jp/

[2] Lingyuan Kong \& Robert G. Parker, Steady mechanics of layered, multiband belt drives used in continuously variable transmissions (CVT), Mechanism and Machine Theory, 43, pp. 171-185, 2008.

[3] Nilabh Srivastava \& Imtiaz Haque, Transient dynamics of metal V-belt CVT: Effects of band pack slip and friction characteristic, Mechanism and Machine Theory, 43, pp. 459-479, 2008.

[4] Jean-Philippe Gauthier \& Philippe Micheau, A model based on experimental data for high speed steel belt CVT, Mechanism and Machine Theory, 45, pp. 1733-1744, 2010.

[5] G. Julió \& J.-S. Plante, An experimentally-validated model of rubber-belt CVT mechanics, Mechanism and Machine Theory, 46, pp. 1037-1053, 2011.

[6] Su-Gwang Jeong, Jeong-Hun Lee \& Jungki Seo, Sumin Kim, Thermal performance evaluation of Bio-based shape stabilized PCM with boron nitride for energy saving, International Journal of Heat and Mass Transfer, 71, pp. 245-250, 2014.

[7] D. Utu a,b, G. Marginean, C. Pogan, W. Brandl \& V.A. Serban, Improvement of the wear resistance of titanium alloyed with boron nitride by electron beam irradiation, Surface \& Coatings Technology, 201, pp. 6387-6391, 2007. 
188 High Performance and Optimum Design of Structures and Materials

[8] S. Mahathanabodee, T. Palathai, S. Raadnui, R. Tongsri \& N. Sombatsompop, Effects of hexagonal boron nitride and sintering temperature on mechanical and tribological properties of $S S 316 \mathrm{~L} / \mathrm{h}-\mathrm{BN}$ composites, Materials and Design, 46, pp. 588-597, 2013.

[9] Gewen Yi \& Fengyuan Yan, Effect of hexagonal boron nitride and calcined petroleum coke on friction and wear behavior of phenolic resinbased friction composites, Materials Science and Engineering A, 425 pp. 330-338, 2006.

[10] Clemens Steinborn, Mathias Herrmann, Uwe Keitel, Andreas Schönecker, Jens Eichler, Correlations between microstructure and dielectric properties of hexagonal boron nitride, Journal of the European Ceramic Society, 2014. 